\section{IS AN ATOMIC MICROSCOPE IN OUR FUTURE?}

Stephen W. Carmichael, ${ }^{1}$ Mayo Clinic

When you read the header to this article, you probably thought I made a mistake. Doesn't he mean atomic force microscope, the familiar AFM? And isn't that microscope in our present, not in our future? Well, l'm not confused. The reference is to a beam of atoms that some day may be used in an atomic microscope, just like a beam of electrons is used in an electron microscope. It would be even more accurate to refer to an "atomic de Broglie microscope," but that's a rather awkward label.

Resolution of any optical microscope (as distinguished from a physical scanning probe microscope, like the AFM) is influenced by the wavelength of the imaging beam; the shorter the wavelength, the better the resolution. An imaging beam of helium atoms offers some distinct advantages. A ground-state thermal energy helium atom has such low energy (5-100 meV) that it is completely non-penetrating and produces practically no mechanical damage. Also, intense beams of the same wavelength (monochromatic) can easily be generated as a supersonic jet. Most importantly, quantum mechanics tells us that a helium atom, like all matter, exhibits wave properties. As first proposed by de Broglie in 1924, any particle has a characteristic wavelength. The de Broglie wavelength of a thermal energy helium atom is only a few Angstroms, much shorter than that of visible light, which promises exceptional resolution if the helium beam can be focused to this limit. But focusing presents a problem. Since the helium atom does not spin and is not very polar, it cannot be focused by standard electromagnetic methods. A collaborative effort organized by Christof W5II, has now demonstrated that a beam of helium atoms may be sufficiently focused to function in a new type of microscope. ${ }^{2}$

The breakthrough was the design and fabrication by Stefan Rehbein and Günter Schmahl of a freestanding Fresnel zone plate to function as a lens in an optical path of helium atoms. A Fresnel "lens" is a concentric grating of interspaced open and opaque annular rings that diffract the waves passing through them. The radii of the annuli were chosen such that all de
Broglie waves diffracting back to the optical axis arrive in phase with each other at one specific point on the axis. The waves add constructively only at this one point, which therefore constitutes a truce focal point for the beam of atoms.

The optical path designed by Bruce Doak, Robert Grisenti, and Peter Toennies worked like this. A beam of helium atoms $\left({ }^{4} \mathrm{He}\right)$ was generated by supersonic free-jet expansion through a nozzle 5 microns in diameter. The jet was directed through a special aperture called a "skimmer", which is a piece of glass capillary tubing that was tapered down to an open end of a few microns. Interestingly, they were assisted in making the skimmers by Ernst Neher, who is famous for developing the patch-clamp, employing identical glass tubes. The skimmers were placed in the pathway to provide a point-like beam source. About a meter down the path, the Fresnel lens was placed, and a slit with a detector was situated about half a meter further down the path. Doak et al. found that the beam was focused down to about $40 \%$ of its original size, to measure about two microns in diameter. Compared to earlier attempts to produce a focused beam of helium atoms using apertures alone, this optical apparatus increased resolution by a factor of ten, signal intensity by a factor of a thousand, and the focused beam density was increased by a factor of a hundred million!

Doak et al. have demonstrated that a beam of helium atoms can be focused. Potentially, such a beam could be fashioned into a device analogous to a scanning electron microscope (SEM), but offering total surface sensitivity and absolutely no sample damage. Not only that, but the scattering of helium atoms from singlecrystal surfaces has been fully explored over the last three decades, allowing for several contrast mechanisms to be exploited. We can easily imagine that a new atomic microscope will open new windows on the atomic landscape!

I The author gratefully acknowledges $\mathrm{Dr}$. Bruce Doak for reviewing this article.

2 Doak, R.B., R.E. Grisenti, S. Rehbein, G. Schmahl, J.P. Toennies, and C. Wöll, Towards realization of an atomic de Broglie microscope: Helium atom focusing using Fresnel zone plates, Physical Review Lett. 83:4229-4232, 1999. See also the report by Andrew Watson, Helium beam show the gentle, sensitive touch, Science 286:1831, 1999.

\title{
I
}

Is An Atomic Microscope In Our Future? .................................... 3 Stephen W. Carmichael, Mayo Clinic

Could You Have Passed The 8th Grade in 1895?

Probably Not. . Take A Look

\section{M\&M 2000 EXPERT'S SESSION ON}

FACILITY MANAGEMENT

Part 3: Justification of Cost, Cost Recovery: Electronic ...........8

Bookkeeping and Billing

Part 4: Concluding Discussion Of Current And Possible ....... 16

Future Topics

Debby Sherman (Organizer), Purdue University

The Microscopy Society of America's Project MICRO:............ 18

The Vermont Experience

Douglas J. Taatjes \& Janet Schwarz, University of Vermont

Telepresence Confocal Microscopy.

J.H. Youngblom, J. Wilkinson \& J.J. Youngblom

California State University-Stanislaus
3 Bugscope: The Second Year Of A Sustainable Remote ........22 Microscope Project For K-12 Educational Outreach

C.S. Potter, B. Carragher, L. Carroll, C. Conway*, B. Grosser, J. Hanlon, N. Kisseberth, S. Robinson, D. Stone*, U. Thakkar** and D. Weber. Beckman Institute, *University High School and ${ }^{*}$ National Center for Supercomputing Applications, University of Illinois

A Pump for Liquid Nitrogen ....................................................24 Gordon Couger, Oklahoma State University (Retired)

LR White Flat Embedding of Cells Grown On Coverslips.......26 Tamara Howard, Cold Spring Harbor Laboratory

A Fool-Proof Method for Mounting Serial Sections .................27 On Single Hole Grids

Debbie Sherman, Purdue University

Odd Vibration Sources 28

Tina (Weatherby) Carvalho, University of Hawaii

NEW PRODUCT NEWS 


\section{McCRONE RESEARCH INSTITUTE}

\section{January - December 2001 Microscopy Courses}

\section{Methods}

Applied Polarized Light Microscopy (1201)

January 15-19 March 12-16 April 23-27

July 9-13 August 27-31 October 22-26

December 10 - 14

Advanced Applied Polarized Light Microscopy (1251*)

April 30 -May 4 October 29 - November 2

Digital and Video Microscopy (1105)

April 16-20 October 15-19

Fluorescence Microscopy (1210)

May 28-30

Microchemical Methods (1270A*)

July $16-20$

Crystal Morphology and Optics (1301)

June 11-15

Particle Isolation, Manipulation and Mounting

for Additional Analysis (1501E)

November 5-9

Fusion Methods and Polymorphism (1260*)

April 9-13

Advanced FTIR Microspectroscopy (1422)

April 9-13 September 10-14

Microtomy Methods in Light Microscopy (1502*)

September 3-5

Conoscopic Techniques for Polarized Light

Microscopy (1310*)

March 19-23

Scanning Electron Microscopy (1402)

April 23-27 November 12-16

\section{Asbestos / Environmental}

Microscopical Identification of Asbestos (1608A)

February 5-9

April 2 - 6

June 4 - 8

August 6 - 10

October 1 - 5

December 3 - 7

Advanced Asbestos Identification (1608B*)

March 5 - 9

November 12 - 16

Indoor Air Quality: Microscopy of Dust, Spores and Pollen (1630)

February $19-23$

September 24 - 28

Asbestos Fiber Counting (NIOSH 582) (1616)

January 29 - 2 Feb. . May $21-25$

October $8-12$

\section{Forensic / Materials}

Forensic Microscopy (1204)

( $\leftarrow$ Same as Applied Polarized Light Microscopy)

Advanced Forensic Microscopy (Trace Evidence) (1701*)

April 30-4 May October $29-2$ Nov.

Forensic Hair and Fiber Microscopy (1207)

May 14 - 18

Microscopy of Illicit Drugs and Excipients (1726*)

July $9-13$

Microscopy of Botanical Traces (1536)

May 7 - 11

Wood and Vegetable Fiber Microscopy (1540)

March $19-23$

\section{Specialities}

Microscopy for Art Conservators (1206)

September 10 - 14

Pharmaceutical Microscopy (1203)

September $17-21$

Polymer Microscopy (1205)

February 26 - March 2

Pollen and Spore Identification (1537)

July $16-20$

Food Microscopy (1560)

July 30 - August 3

Microscopy of Crystal Caking Problems (1760)

September 24-26

*Prerequisites: course 1201 (PLM) or consent of instructor;

1608 A for $1608 B$

To receive detailed information contact Nancy B. Daerr, McCrone Research Institute, 2820 S. Michigan Aveue, Chicago, IL 60616-3292

PHONE: $312 / 842 \bullet 7100$

FAX: $312 / 842 \bullet 1078$

e-mail: ndaern mcri.org

Visit our Home Page on the World Wide Web at:

http://www.mcri.org 


\section{Project MICRO}

Microscopy Today (MT) is a magazine for professional microscopists, and the Microscopy Society of America (MSA) is a professional society for microscopists. Both provide microscopists with much useful information. Why then is Microscopy Today's mailing list more than twice MSA's membership? Half of MT's readership is missing out on the support that MSA provides! This issue of Microscopy Today was produced in collaboration with MSA's Project MICRO; it's a small sample of the information that MSA offers its membership. Microscopy education at both precollege and university levels are featured; two articles are reprints of abstracts of papers about remote access microscopy that were presented at MSA's annual meeting in Philadelphia last August, and a third describes a Vermont middle school outreach program supported by MICRO. MICRO's newly revised bibliography, mailed with this issue, is MSA's gift to you. If you can't use it yourself, PLEASE give it to a teacher, or a parent, or a Scout leader, or a librarian-anyone who can use it to introduce children to the microworld. But first, photocopy the last page and use it to give yourself a MSA membership.

... Caroline Schooley, Project MICRO Coordinator schooley@men.org

Editor's Note: As it happens, I am now in my 21st year as a member of the Microscopy Society of America-and what a pleasure, both professional and personal, it has been. And while I have not met many other members, through their work in the Society, I have found them to be kindred souls.

If you are not a MSA member, I most strongly recommend that you become one. You will find that the modest $\$ 45$ yearly membership fee is worth much to you in your profession. As noted, a membership application is on the last page of the enclosed Bibliography.

\section{M\&M 2000 Expert's Session On}

\section{Facility Management}

In the two previous issues of this publication we presented Part 1 and Part 2 of the Expert's Session On Facility Management as discussed at M\&M 2000 recently in Philadelphia. In this issue we have presented the remaining two parts: Part 3 Justification of Cost, Cost Recovery, Electronic Bookkeeping and Billing (Page 8) and Part 4 Concluding Discussion of Current and Possible Future Topics (Page 16).

Suggestions for future topics will be considered for discussion at a similar Experts Session on Facility Management in conjunction with M\&M 2001 at Long Beach, CA. If you have topic suggestions, please send them to Debby Sherman at: dsherman@purdue.edu . They must be submitted by January 2001 to be considered.

Halcyonics GMbH announces the Accurion LLC will be distributing Halcyonics' anti-vibration equipment exclusively in the U.S. and Meiwa Shoji Co. Ltd. Will do the same in Japan. Halcyonics' anti-vibration equipment actively eliminates a wide range of vibrations for small and large instrumentation. Accurion LLC: (510)445-1533
The Microscopical Society of Canada will be holding its 28th Annual Meeting at The University of New Brunswick in Fredericton, New Brunswick, Canada on June 6/8, 2001. This annual meeting is devoted to all aspects of microscopy and microanalysis. Some planned topics at the 28th AGM are the contributions of microscopy in the environmental sciences, cryoSEM techniques as applied to plant pathogenic fungi, EELS in TEM and Confocal Laser Scanning Microscopy. In addition to the platform and poster sessions there will be workshops on specimen preparation in the materials and biological sciences and on digital image techniques. The deadline for abstracts is 30 March 2001.

Incidentally, Fredericton is an easy eight hour drive from Boston and a two hour flight from Toronto.

For further information, visit their web site at <www.unb.ca/ msc2001> or contact the Program Chair, Dr. Joseph White at (506)447-3185 or clancy@unb.ca

\section{MAS Special Topics Workshop}

The NIST Microanalysis Research Group and the Microbeam Analysis Society will co-sponsor an MAS Special Topics Workshop, "Understanding the Accuracy Barrier in Quantitative Electron Probe Microanalysis and the Role of Standards" that will be held at NIST in Gaithersburg, Maryland on October $15 / 18,2001$. This event is part of the 2001 celebration of the NIST Centennial. The limited attendance workshop will deal with the experimental and theoretical factors that currently limit the accuracy of quantitative results to approximately $2 \%$ relative. The topics will include sample and instrument parameters, counting statistics, correction procedures, individual matrix correction parameters, etc. In addition, one or more sessions will be specifically devoted to discussions on microanalysis standards. Invited speakers will be expected to provide a short (6 page, maximum) manuscript of their paper at the time of the meeting. The intent of the meeting and monograph is to provide detailed information on accuracy limits and on acceptable standards procedures in quantitative microprobe analysis that is presently not readily available in the literature.

We are now preparing a list of invited speakers, and we are actively soliciting volunteers who would like to be considered to present a talk, especially on microanalysis standards. And, if you are interested in attending the meeting contact:

Dale Newbury: (301)975-3921, dale.newbury@nist.gov

Ryna Marinenko@nist.gov: (301)975-3901,

ryna.marinenko@nist.gov

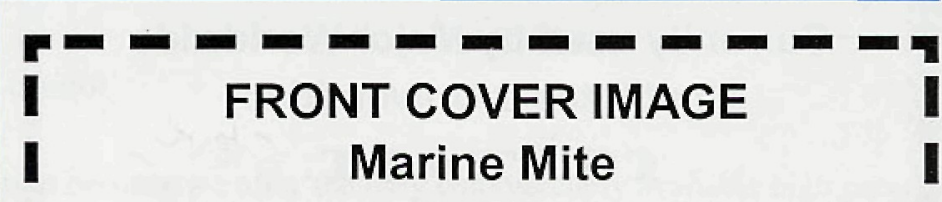

The marine mite is a predacious mite, one of many species in the family of Marine Meiofauna. These are critters under $1 \mathrm{~mm}$ in size that live between the sand grains at the bottom of the ocean and to the beaches.

Magnification: Approximately $2,750 \mathrm{X}$

Copyright@ by David Scharf, 2000, All Rights Reserved 